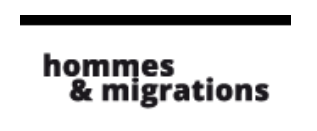

Hommes \& migrations

Revue française de référence sur les dynamiques

migratoires

$1283 \mid 2010$

Cuisines et dépendances

\title{
L'adaptation des migrants russes aux pratiques alimentaires à Paris et à Londres
}

Du basculement alimentaire au basculement identitaire?

\section{Olga Bronnikova et Maria Emanovskaya}

\section{(2) OpenEdition}

1 Journals

\section{Édition électronique}

URL : http://journals.openedition.org/hommesmigrations/993

DOI : 10.4000/hommesmigrations.993

ISSN : 2262-3353

Éditeur

Musée national de l'histoire de l'immigration

\section{Édition imprimée}

Date de publication : 1 janvier 2010

Pagination : 74-85

ISBN : 978-2-919040-04-9

ISSN : 1142-852X

Référence électronique

Olga Bronnikova et Maria Emanovskaya, "L'adaptation des migrants russes aux pratiques alimentaires à Paris et à Londres », Hommes \& migrations [En ligne], 1283 | 2010, mis en ligne le 29 mai 2013, consulté le 01 mai 2019. URL : http://journals.openedition.org/hommesmigrations/993 ; DOI : 10.4000/hommesmigrations.993 
Du basculement alimentaire au basculement identitaire?

\section{L'adaptation des migrants russes aux pratiques alimentaires à Paris et à Londres}

Par Olga Bronnikova, doctorante en géographie, institut national des langues et civilisations internationales, MIGRINTER, CNRS-université de Poitiers

Par Maria Emanovskaya, doctorante en géographie (ED434, Ecole doctorale de géographie de Paris)

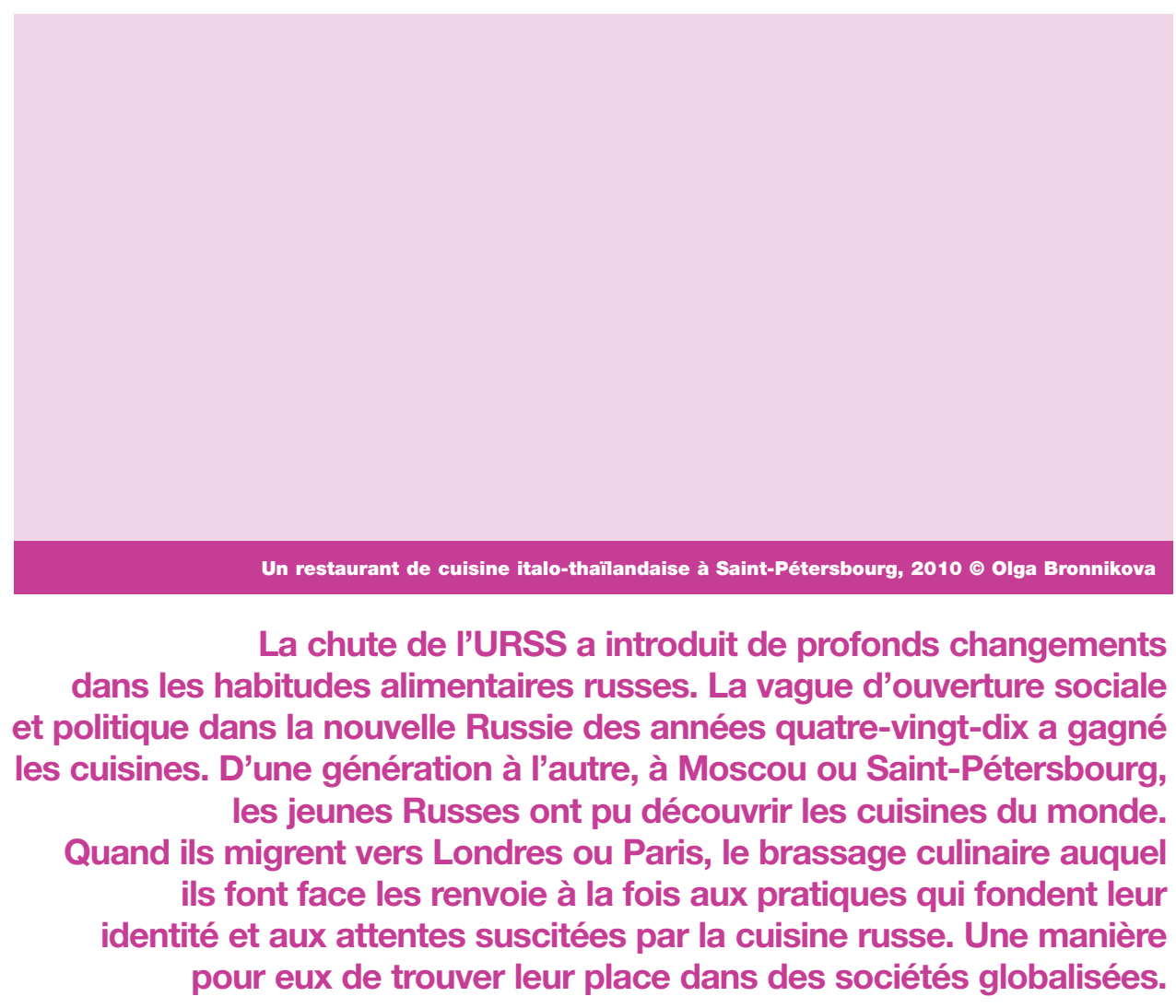


La particularité des pratiques de consommation dans les pays postsoviétiques se trouve sans aucun doute dans leur évolution rapide. Les anthropologues y ont trouvé un champ fructueux d'investigation dans les façons dont les citoyens négocient et s'approprient les changements sociaux, économiques et politiques à l'échelle du pays. L'alimentation constitue un prisme fascinant pour tracer et mesurer des trajectoires diverses, inattendues, curieuses et souvent paradoxales, ainsi que les conséquences des transformations qui ont lieu sur le territoire de l'exURSS et de l'Europe de l'Est depuis plus d'un siècle. "L'alimentation est un canal particulièrement conducteur pour mettre en cuvre et comprendre le changement social, à la fois parce que sa matérialité en fait un marqueur concret de transformation et parce que les qualités sensuelles de la nourriture suscitent des réponses viscérales qui transforment les processus sociaux externes et anonymes en expériences personnelles intimes et immédiates. La nourriture ne rend pas seulement compte de la dimension politique de la personnalité, mais elle rend également le monde accessible aux personnes ordinaires d'une manière différente des autres $^{(1)}$."

Centrer la recherche sur les migrations postsoviétiques nous confronte à la réalité mouvante de la Russie

L'ouverture qui a suivi la chute de I'URSS a apporté d'autres aliments et recettes auparavant inconnus. Ainsi, les habitudes alimentaires au moment du départ ne sont guère issues uniquement de la cuisine "russe". toujours changeante, ouverte depuis peu à la mondialisation et en quête d'une nouvelle identité. Il s'agit d'appréhender des habitudes alimentaires en situation de migration et non la cuisine russe, et ce pour plusieurs raisons. Premièrement, le métissage culinaire est important en Russie. Historiquement, avec l'incorporation des plats issus des cuisines de tous les coins de l'URSS, le plov, le chachlik sont devenus les plats les plus communs des tables russes. L'ouverture qui a suivi la chute de l'URSS a apporté d'autres aliments et recettes auparavant inconnus. Ainsi, les habitudes alimentaires au moment du départ ne sont guère issues uniquement de la cuisine "russe".

Deuxièmement, les habitudes alimentaires connaissent des transformations profondes en situation d'immigration: d'un côté, la rencontre et l'intégration progressive de nouvelles façons de se nourrir (aliments, techniques de cuisson, recettes) et, d'un autre côté, le repli sur la cuisine nationale en tant que moyen de préserver et transmettre une identité. Cependant, la vision que les migrants ont de leur cuisine nationale subit des changements à leur arrivée. Elle est influencée par l'image que cette cuisine a dans la société d'accueil, par l'offre de commerces alimentaires ethniques et par les attentes des invités à la table des migrants. 


\section{Pour une méthodologie aussi mouvante que le processus étudié ?}

Cet article se fonde sur une enquête effectuée auprès d'une vingtaine de Russes habitant la région parisienne et d'une autre vingtaine habitant le Grand Londres. Il s'agit d'un échantillon composé de jeunes hommes et de jeunes femmes russes décidés à migrer après la chute de l'URSS et souvent motivés par une recherche d'emploi en France ou en Grande-Bretagne. Tous les individus interviewés sont arrivés relativement récemment (en 1998, au plus tôt). La majorité est partie après les années deux mille. L'échantillon a été choisi en fonction de questionnements sur le changement des pratiques alimentaires, lié aux conditions d'immigration et à l'évolution du contexte socio-économique et politique en Russie après les années quatre-vingt-dix. Les questions de l'enquête ont porté sur deux dimensions, suivant une optique comparative : les habitudes alimentaires avant le départ et l'imaginaire alimentaire - les attentes par rapport à la cuisine française et britannique avant le départ, les attentes des invités issus de la société d'accueil vis-à-vis de la cuisine russe et le regard extérieur sur les évolutions actuelles du paysage alimentaire russe. En outre, cette enquête traite de manière indirecte de la sociabilité et de l'inté-

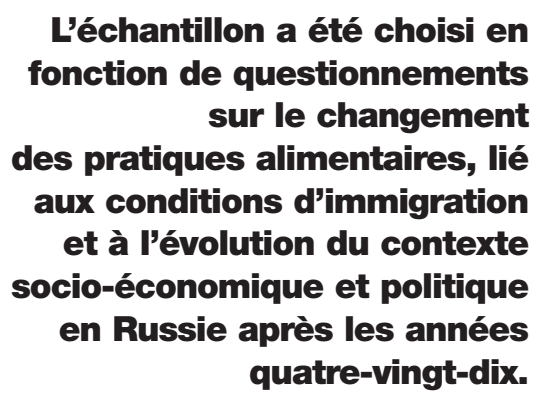
gration dans la société d'accueil des migrants russes.

La "nouvelle" génération de Russes nés dans les années quatre-vingt nous intéresse particulièrement en tant que représentante de cette nouvelle Russie qui traverse une période de recherche identitaire. Cependant, dans une optique de comparaison, nous avons mis au point quelques questionnaires et procédé à des entretiens exploratoires auprès de jeunes hommes et de jeunes femmes installés à Paris et à Londres, nés dans les années soixante-dix et partis de Russie à la fin des années quatre-vingt-dix et au début des années deux mille. Cette démarche nous a permis de mettre en évidence des différences intergénérationnelles en terme d'héritage culturel. Autrement dit, nous avons voulu démontrer l'existence de différences dans les pratiques alimentaires de deux groupes d'âges: d'une part, des individus ayant entre 30 et 35 ans, nés et ayant grandi en URSS, mais qui ont également vécu dans la Russie nouvelle, et, d'autre part, des individus entre 25 et 30 ans, nés en URSS et ayant grandi en Russie. Cette démarche volontaire a révélé 
plusieurs points communs, notamment dans les habitudes alimentaires à domicile entre les deux groupes. Mais elle a également mis au jour des différences dans la connaissance d'autres cuisines que la cuisine russe et au niveau des prises de repas dans les établissements de restauration. En effet, dans les deux groupes cités, l'héritage culturel soviétique est présent, mais à des degrés variés.

\section{Dis-moi ce que tu manges et je te dirai si tu es Soviétique ou Russe}

Les différences intergénérationnelles sont importantes dans le cas d'une Russie en pleine évolution. Il nous est donc paru intéressant de questionner les projets migratoires, mais aussi professionnels, de ces migrants russes.

Tous ces individus sont diplômés et appartiennent le plus souvent à ce qu'on appellera ici des "migrations professionnelles". Cependant, derrière ce terme se cachent des trajectoires migratoires singulières. Certains migrants sont partis de Russie pour leurs études et sont restés travailler à Paris ou Londres ; d'autres sont arrivés pour leur travail ou encore pour un mariage. Cependant, tous forment un groupe homogène d'un point de vue professionnel. C'est-a-dire que la majorité écrasante travaille dans les domaines de l'expertise (notamment écologique), des finances et des hautes technologies. Ceci peut s'expliquer par la structure des marchés du travail des villes globales qui "concentrent désormais des ressources immenses par le truchement d'activités tertiaires supérieures dont l'influence est de plus en plus déterminante sur leur système économique et social ${ }^{2}$ ". Selon l'hypothèse de S. Sassen, les villes mondiales ne sont pas uniquement les centres, les nceuds des transactions internationales, mais aussi des lieux de production des services de direction de "réseaux géographiquement éclatés d'usines, de bureaux et de services extérieurs". Elles sont également des lieux où se développent la "production de nouveautés financières et la construction de marchés, toutes deux étant centrales à l'internationalisation et à l'expansion de l'industrie financière $e^{(3)}$ ". Cependant, elle précise qu'à ces critères ne correspondent que trois villes: Londres, New-York et Tokyo.

Selon S. Sassen, Paris peut être appelée "ville globale soft", car une marge important de sa population appartient à la classe moyenne, ce qui n'est pas le cas des villes globales par excellence. L'échantillon de notre enquête (quarante entretiens à Paris et trente-cinq entretiens à Londres) effectuée dans le cadre d'une thèse a confirmé que la part des personnes hautement qualifiée, cette "élite transnationale" dans la 
population russe immigrée, est plus importante à Londres qu'à Paris. Néanmoins, nous ne pouvons pas vérifier ce fait avec exactitude, car notre échantillon reste relativement réduit à cause de son caractère qualitatif.

\section{Au-delà de la mobilité géographique}

De même, le choix des interviewés a été motivé par notre problématique de départ qui cherche à comprendre la mobilité de l'alimentation. "Des fast-foods aux cuisines des migrants, des cybercafés aux livraisons à domicile, l'alimentation circule... Les villes bougent, les populations bougent, la nourriture bouge : suivre à la trace ces mouvement crée une nouvelle attention sur les géographies culinaires urbaines, sur les villes comme

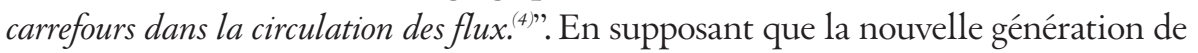
Russes soit plus mobile que l'ancienne, notre choix a porté sur ces nouveaux citoyens russes qui migrent vers la France ou la Grande-Bretagne, voire qui circulent entre l'un de ces deux pays et la Russie.

La provenance géographique de nos migrants se limite à deux villes russes : Moscou et Saint-Pétersbourg. Néanmoins, ceci ne veut pas dire que ces migrants soient originaires de ces villes. Le plus souvent, il y a eu précédemment une histoire migratoire dans leur vie : plusieurs sont arrivés à Moscou pour leurs études ou leur travail, avant de partir en France ou en Grande-Bretagne.

Deux vagues de départ sont à distinguer : celle des années quatre-vingt-dix et celle de la décennie deux mille. Elles diffèrent selon les motivations de départ et les attentes des migrants vis-à-vis de leur cadre de vie à leur arrivée. Mais le pays de départ est également différent. Ici seront présentées deux Russies différentes selon leur héritage alimentaire.

La décennie suivant la chute de l'URSS est profondément marquée par un intérêt pour l'Occident hérité de la période soviétique ${ }^{(5)}$. Séparés de l'Occident par le rideau de fer durant des dizaines d'années, les Soviétiques ont appris à considérer les biens venus d'Occident comme a priori attractifs et désirables. En même temps, l'industrie du pays s'écroule et la situation sur le marché alimentaire suit cette tendance générale. Le bonheur à la russe par deux gastronomes en exil ${ }^{(6)}$ donne un aperçu des nostalgies culinaires de cette émigration : "Des crabes d'Extrême Orient russe, des melettes épicées de Talline, des gâteaux aux gaufres 'pralinées', du chocolat comme 'Mishka na severé', de l'eau minérale Iessentouki (de préférence numéro 17). Avec un tel assortiment (ah oui, de la moutarde russe forte), la vie à l'étranger (et de l'huile de tournesol pressée à chaud) devient meilleure (de tomates marinées aigre-doux) et plus gaie (le congnac Ararat, six étoiles)." 
La crise de 1998 a profondément bouleversé l'ordre établi. L'industrie alimentaire a profité de la hausse des prix des produits importés et a su reconquérir le marché. Cependant, il ne faut pas voir dans cette décennie une parenthèse pour le système alimentaire russe. Nombreux sont les aliments qui y sont entrés d'une façon plus ou moins définitive, de même que les modes de consommation dont nous ne citons ici que quelques exemples.

Les fast-foods sont profondément ancrés dans les pratiques de consommation courante. McDonald's, dont l'offre s'est banalisée, se voit concurrencé

par de nombreuses chaînes d'a-

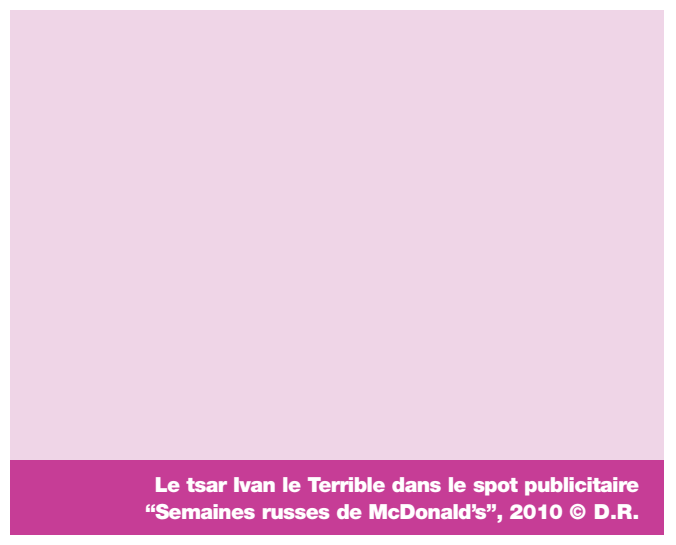
limentation rapide à la russe.

Ces dernières réinventent et ajustent les traditions culinaires de la Russie tsariste. Pour le vingtième anniversaire de l'ouverture du premier McDonald's en Russie, l'entreprise a lancé les Semaines russes : hamburger au pain de seigle, pirojki aux champignons, tandis que le spot publicitaire avec Ivan le Terrible ${ }^{(7)}$ associait ce fast-food aux traditions ancestrales (cf. photographie ci-dessus).

Les sushis sont omniprésents dans les plus grandes villes russes. En plus de nombreuses chaînes de restaurants japonais, ils sont sur la carte de la plupart des restaurants de "cuisine européenne", de cuisine fusion, etc.

\section{De la cantine au restaurant: une ouverture aux cuisines du monde}

Les pratiques alimentaires ne sont pas les mêmes dans les villes de province russe et dans les grandes métropoles comme Saint-Pétersbourg et Moscou. Les différences concernent les modes d'alimentation (seul ou avec amis/famille/compagne) et les lieux de consommation. Il est intéressant de noter que nos interviewés qui sont arrivés à Moscou depuis les villes de province disaient ne pas fréquenter d'établissements de restauration (à l'exception des cantines universitaires), manger de manière non régulière et ne pas faire trop de distinction entre les différentes cuisines. Ce mode d'alimentation était caractéristique lors de leur séjour à Moscou. 
"À cette époque [les années quatre-vingt-dix], quand jétais étudiant à Moscou, on mangeait principalement dans la cantine universitaire. Le plat le plus commun était le sossiska v teste ${ }^{(8)}$ avec une tasse de thé. Du coup, quand je rentrais chez mes parents à Kalouga, je mangeais bien, même si on n'était pas riche." (D., 23/12/2009)

Ainsi, ce mode d'alimentation est commun aux étudiants, et notamment aux étudiants de la période soviétique. Nos interviewés qui appartiennent à la tranche d'âge des 30-35 ans et qui sont partis à la fin des années quatre-vingt-dix ont découvert en France ou en Grande-Bretagne des cuisines du monde : indienne, asiatique, chinoise, japonaise, coréenne, thaïlandaise, italienne et, bien évidemment, française ou britannique. Avant leur

\section{Les migrants âgés entre} 20 et 25 ans fréquentaient souvent ou très souvent les établissements de restauration en Russie et continuent de les fréquenter dans le pays d'arrivée en découvrant des cuisines du monde.

départ, ils ne connaissaient que la cuisine russe et quelques plats des cuisines des pays postsoviétiques (plov ouzbek, chachlik caucasien et plats ukrainiens). Nos interviewés fréquentaient peu ou pas les établissements de restauration.

Nous voudrions insister sur ce point, car la situation n'est plus vraiment la même avec la génération née dans les années quatrevingt. "En particulier, le domaine du nouveau consommateur en Russie, tel qu'il se manifeste dans une culture florissant de restaurants, cafés et épiceries, a produit de nouvelles formes d'espace public qui, à leur tour, présentent des opportunités pour les consommateurs russes, générant de nouveaux comportements et de nouvelles idéologies sur ces comportements. ${ }^{(9) "}$ Les migrants âgés entre 20 et 25 ans fréquentaient souvent ou très souvent les établissements de restauration en Russie et continuent de les fréquenter dans le pays d'arrivée en découvrant des cuisines du monde.

\section{Regards sur soi et perception des changements}

Malgré le brassage culinaire que les Russes ont connu avant leur départ, une véritable découverte gustative les attendait à leur arrivée en France ou en GrandeBretagne.

Tous les interviewés retournent régulièrement - une à deux fois par an - en Russie et ont la possibilité de suivre l'évolution des comportements alimentaires de leurs compatriotes. Ils mentionnent l'accroissement du choix des établissements de 
restauration et de produits d'alimentation dans les commerces. Ils évoquent, cependant, l'augmentation des prix qui atteignent parfois le niveau européen, alors que les produits restent inférieurs du point de vue qualitatif. En ce qui concerne l'alimentation à domicile, ils notent que celle-ci reste la même et qualifient cette cuisine de "grasse". Il est important d'évoquer ce fait du point de vue du changement des préférences gustatives des migrants. Plusieurs ont mis du temps pour s'habituer à la cuisine française, mais ont commencé à "beaucoup l'apprécier" avec le temps. "Je ne m'attendais pas du tout à voir des boudins, surtout des boudins noirs faits de sang cuit. La tête de veau m'a beaucoup troublé aussi... J'imaginais plutôt les cuisses de grenouille sous toutes les sauces." (L., 14/12/2009)

Tous ont signalé un changement dans leurs pratiques alimentaires. Ils disent consommer davantage de fruits et de légumes, notamment des salades. Ils estiment globalement que leur alimentation est devenue plus régulière (trois fois par jour à heures fixes) et plus saine. Il est possible que ce mode d'alimentation soit dû aux campagnes de promotion de modes de vie et d'alimentation plus équilibrés. Nos migrants, qualifiés, citadins, jeunes, font partie de la population la plus sensible à ce discours et ouverte aux influences extérieures.

\section{L’expérience du métissage culinaire...}

L'exemple du métissage culinaire (voir encadré p. 82) est retranscrit dans les pratiques à domicile. Toutes les personnes interviewées (qui cuisinent à domicile) ont signalé la variété des plats et des recettes qu'elles utilisent aujourd'hui. Elles qualifient leur alimentation à domicile de "très métissée" et se tournent vers des choix de plats à la fois exotiques et équilibrés. "Je ne peux pas décrire en deux mots la cuisine que je prépare chez moi. Le lundi, je fais un plat au curry. Le mardi, je prépare un risotto. Le mercredi, on mange une salade et le jeudi, du sarrasin avec des harengs. Je ne trouve pas une caractéristique unique de ma cuisine. Peut-être je peux la qualifier de cuisine internationale." (N.,15/12/2009)

Le choix des restaurants non plus ne se limite pas à la cuisine française. Tous fréquentent régulièrement des restaurants de cuisine asiatique, indienne, maghrébine ou africaine. Ils se disent, de même, clients des épiceries alimentaires "exotiques", notamment chinoises et indiennes, pour leur cuisine à domicile.

Si l'alimentation à domicile est très métissée, la cuisine du pays d'accueil est aussi présente dans le régime alimentaire des migrants russes. Les résidents à Paris indiquent que leur déjeuner a lieu pendant les heures de travail, avec des collègues français, dans les brasseries ou restaurants de cuisine française. Certains interviewés ont signalé 
Le métissage alimentaire,

un modèle de Jean-Pierre Poulain et Laurence Tibère ${ }^{(11)}$

La mondialisation "implique à la fois des nouvelles différenciations, résultant des formes originales d'appropriation de produits ou de techniques, et le développement d'espaces communs servant de passerelle entre les modèles alimentaires ${ }^{(12) !}$. Ainsi, les restaurants exotiques "constituent un espace intermédiaire, en même temps lieu d'initiation, sas, première étape vers une découverte ultérieure plus précise de la culture alimentaire" en question. Ils permettent le cheminement de cette dernière vers l'univers gastronomique du pays d'accueil. "De cet espace alimentaire original naissent toute une série de plats ou de pratiques qui n'existent dans aucune des deux gastronomies ${ }^{(13) .}$."

L'exemple réunionnais "met en évidence trois grands mécanismes concomitants de rencontres des systèmes alimentaires : un mouvement d'intégration, inscrit dans les processus de créolisation, des jeux de différenciation qui traduisent la réaffirmation des particularismes communautaires, et enfin un espace d'échanges, d'adoption et d'influences réciproques, en particulier entre l'espace créole et les autres systèmes alimentaires réunionnais ${ }^{(14) !}$.

Les espaces alimentaires (espace de mise en commun, mais aussi espace d'opposition) traduisent les mouvements d'une dialectique d'intégration/différenciation qui met en jeu différentes formes d'échange, "d'acceptation ou de rejet d'une pratique sur des logiques d'équivalence fonctionnelle ou d'équivalence symbolique ${ }^{(15) !}$. L'adoption se fait plus facilement si les cultures alimentaires locales disposent d'un ensemble de recettes et d'usages socialement valorisés, susceptibles de s'appliquer au nouvel aliment.

Le concept de créolisation est, par conséquent, préférable à celui d'acculturation de fait de sa "capacité à poser les résultats du métissage comme un nouveau tout organisé et non comme des résidus de processus de décomposition ${ }^{(16) !}$.

avoir voyagé en France pour des séjours-découvertes de restaurants étoilés. Les Londoniens ont à peine mentionné la cuisine britannique traditionnelle, seulement dans la partie de leurs attentes et de leurs connaissances précédant leur départ.

\section{... et de la nostalgie}

Ils restent tout de même nostalgiques de la cuisine russe. À ce sujet, ils mentionnent l'évolution de leurs préférences en matière de produits russes. Au tout début ils se disaient nostalgiques des salades traditionnelles russes (salade "Olivier", ou Stolichniy) assaisonnées de grandes quantités de mayonnaise. Aujourd'hui, le "mal du pays" est évoqué à travers des produits tels que les cornichons salés, le bortsch (soupe de bettrave) et les pirogy au choux. 
Étant donné que la France est un pays d'accueil historique des migrants russes de différentes époques, la nouvelle vague d'immigration russe a pu trouver sur place une cuisine russe. Mais un décalage profond a pu exister entre cette dernière, qui s'est développée en opposition à la Russie soviétique, et les habitudes alimentaires des migrants. Pour répondre à la nouvelle demande, de nouveaux commerces ont vu le jour. Il ne s'agit pas de restaurants, mais bien d'épiceries dont l'offre est constituée des ingrédients manquant dans d'autres commerces ${ }^{(10)}$. La chaîne des épiceries Gastronom, qui sont fréquentées par nos interviewés, en est un exemple. De nos jours, le réseau des commerces alimentaires russes ou issus de l'espace postsoviétique est largement développé et ancré dans le paysage urbain parisien. Contrairement à Paris, le réseau des commerces alimentaires à Londres s'est développé relativement récemment, durant les deux dernières décennies. Néanmoins, le nombre d'épiceries russes à Londres (soixante) est supérieur à celui de Paris. L'immigration russe en Grande-Bretagne n'a débuté qu'à la fin des années quatre-vingt, mais est vite devenue quantitativement importante. Nous ne pouvons que nous étonner de la rapidité avec laquelle s'est établie la communauté russe à Londres, avec toutes sortes de conséquences. Le commerce ethnique russe en fait partie.

La fréquentation des épiceries, surtout à Paris, reste limitée en moyenne à une fois tous les deux ou trois mois. "Sincèrement, on n'a pas besoin de produits russes pour faire de la cuisine à la maison. Nous utilisons des produits français pour faire des plats russes. On achète juste des pelmenis [raviolis russes] chez Gastronom, car c'est trop compliqué à faire." (D. et O., 1/12/2009) Le besoin d'acheter ces produits russes dans les commerces spécialisés se fait sentir aussi bien pour l'alimentation quotidienne que pour des occasions particulières.

\section{De la cuisine russe pour les autres}

Les migrants russes signalent qu'ils ont organisé à plusieurs reprises des repas pour leurs amis ou collègues français dans l'objectif de leur faire découvrir la cuisine russe. Parmi les produits que les Français et les Britanniques préfèrent, nos interviewés mentionnent le même assortiment de produits (bortsch, pirogy, etc.) en plus de la vodka, du caviar et des ceufs de saumon servis avec des crêpes russes. Cette concordance entre les goûts de nos interlocuteurs et de leur société d'accueil confirme notre hypothèse : les Russes s'adaptent avec le temps aux préférences gustatives de leur pays d'accueil, mais aussi à la vision préexistante en France ou en Grande-Bretagne de ce qu'est la cuisine russe. 
Ainsi se dessine une image très métissée des pratiques alimentaires des migrants russes à Paris. Ils adhèrent aux tendances communes chez les habitants des villes globales - l'alimentation métissée et variée -, mais ils restent tout de même attachés à la cuisine russe et plus précisément à certains plats ou encore produits de l'alimentation russe.

\section{Pour ne pas conclure...}

L'étude du mouvement des pratiques alimentaires donne des indications sur la structure des interactions que les migrants entretiennent avec la société de départ et la société d'arrivée. Autrement dit, la question identitaire se pose inévitablement. Cette enquête nous a permis, premièrement, de distinguer des processus selon les périodes de migration. Les plus jeunes de nos migrants connaissaient avant leur départ l'alimentation "hybride", dans les restaurants de cuisine italo-thaïlandaise en Russie, par exemple. Ils l'adaptent facilement à leur alimentation à domicile en France et en Grande-Bretagne. Les migrants âgés de 30 à 35 ans et n'ayant connu que peu ou pas du tout le boom de la restauration en Russie découvrent les différentes cuisines dans leur pays d'accueil. Cependant, ils suivent également la logique de métissage culinaire à domicile. Ce fait nous montre que le facteur temps ne joue pas de rôle crucial dans l'adaptation aux modes d'alimentation dans les villes globales. L'intérêt pour l'exotisme et l'interculturel naît dans la Russie actuelle, ce qui était impensable en URSS où les différences culturelles étaient nivelées par l'idéologie égalitariste.

Deuxièmement, les deux groupes appartiennent à la même catégorie socioprofessionnelle à Paris et Londres, ce qui n'était pas le cas avant leur départ. Certains de nos interviewés sont issus de milieux sociaux modestes et n'ont atteint le niveau de vie de cadres que dans leurs pays d'accueil. Cet élément doit être pris en compte lorsque les interviewés évoquent le basculement de leur régime alimentaire vers une nourriture plus saine. Le slogan officiel "Manger cinq fruits et légumes par jour!' est incorporé avant tout par la population en mesure de se le permettre, c'est-a-dire par la population citadine relativement aisée, et se présente comme un signe de distinction sociale. Le facteur géographique joue également un rôle moteur. Même si la tendance générale des villes globales est à la consommation rapide pour suivre le rythme de vie citadin, l'“élite transnationale", pour reprendre le terme de S. Sassen, promeut des modes d'alimentation équilibrés.

Troisièmement, malgré l'accélération de la mobilité humaine et des processus de banalisation des cultures, nos migrants cherchent à s'adapter à la ville globale en y 
inscrivant leurs origines. Autrement dit, ils continuent de consommer des plats et des produits russes en les adaptant à la logique d'alimentation des couches aisées parisiennes ou londoniennes. Il leur est également important de faire découvrir la cuisine, et la culture russe, aux Français et aux Britanniques par l'intermédiaire de repas conviviaux.

En définitive, il nous paraît important de soulever la notion d'"étrangeté" à propos des migrants russes, du point de vue de leurs pratiques alimentaires, à l'égard à la fois de leur société d'origine et de la société d'accueil. Ils ne se nourrissent ni comme des Français ou des Britanniques, ni comme des Russes de Russie.

\section{Notes}

1. Melissa L. Caldwell, "Introduction. Food and Everyday Life after State Socialisme", in Melissa Caldwell L.(Éd.), Food and Everyday Life in the Post-Socialist World, Indiana University Press, Bloomington, 2009, p. 3.

2. Saskia Sassen, La ville globale : New York, Londres, Tokyo, Descartes, Paris, 1996, p. 9.

3. Saskia Sassen, op. cit., p. 36.

4. David Bell, "Fragments for a New Urban Culinary Geography", in Journal for the Study of Food and Society, vol. 6, $\mathrm{n}^{\circ} 1,2002$, p. 11.

5. Caldwell évoque le ratio de deux à un caractérisant la préférence du consommateur russe pour les aliments importés par rapport à la production nationale. Voir Melissa L. Caldwell, "The Taste of Nationalism: Food Politics in Postsocialist Moscow", in Ethnos, vol. 67, n³, 2002, pp. 295-319.

6. Petr Vaïl, Aleksandr Guenis, Le bonheur à la russe par deux gastronomes en exil, Monaco, Éd. du Rocher, 2004.

7. Le costume du tsar ainsi que la musique sont issus d'une comédie soviétique culte.

8. Le hot-dog russe, vendu souvent dans les boulangeries soviétiques, qui se mange sans ketchup.

9. Melissa L. Caldwel, "Tempest in a Coffee Pot. Brewing Incivility in Russia's Public Sphere", in Melissa L. Caldwell

(Éd.), Food and Everyday Life in the Post-Socialist World, Bloomington, Indiana University Press, 2009, p. 104.

10. Emmanuel Ma Mung, "L'expansion du commerce ethnique : Asiatiques et Maghrébins dans la région parisienne", Revue européenne des migrations internationales, vol. 8, n 1, 1992, pp. 39-59.

11. Jean-Pierre Poulain et Laurence Tibère, "Mondialisation, métissage et créolisation alimentaire. De l'intérêt du 'laboratoire' réunionnais", Bastidiana \#31-32, juillet-décembre 2002, pp. 225-241

12. Idem, p. 232.

13. Idem, ibidem

14. Idem, p. 235.

15. Idem, p. 238.

16. Idem, p. 239. 